\title{
SOBREPESO E OBESIDADE EM MULHERES NO CLIMÁTERIO EM UM MUNICÍPIO DO NOROESTE DO ESTADO DO PARANÁ
}

Robsmeire Calvo Melo Zurita ${ }^{1}$, Edilaine da Silva Jacom², Rebeca Melo Zurita ${ }^{3,}$ Rosana Rosseto de Oliveira', Emiliana Cristina Melo'

${ }^{1}$ Enfermeira. Doutoranda da Pós Graduação de Enfermagem da Universidade Estadual de Maringá (UEM). ${ }^{2}$ Enfermeira Centro Universitário de Maringá -CESUMAR. ${ }^{3}$ Acadêmica de Medicina. Universidade Federal do Mato Grosso (UFMT). E-mail: robszurita@bol.com.br

\section{RESUMO}

O climatério é fenômeno fisiológico decorrente do esgotamento dos folículos ovarianos que ocorre em mulheres de meia idade. Estudos mostram que o índice de massa corporal (IMC) tem atingido seus valores acima na faixa etária compreendida dos 50 a 59 anos período da menopausa. A obesidade é definida como aumento de depósito de triglicérides nas células adiposas, que sofrem alterações no perfil metabólico que modificam as composições do tecido adiposo. Este estudo teve por objetivo identificar a prevalência de sobrepeso e obesidade nas mulheres no climatério em Maringá no estado do Paraná. Participaram 100 mulheres de 45 a 69 anos. O peso corporal foi avaliado com base no IMC. Trata-se de um estudo quantitativo, analítico e descritivo. Da população estudada $42 \%$ das mulheres estavam obesas, $40 \%$ apresentaram sobrepeso. É fundamental acompanhamento sistemático das mulheres visando à promoção da saúde, diagnóstico precoce, tratamento imediato dos agravos e prevenção de danos.

Palavras-chave: Climatério, enfermagem, índice de massa corporal, obesidade abdominal, serviços de saúde da mulher.

\section{INTRODUÇÃO}

De acordo com Ministério da Saúde (MS) o climatério não é uma doença e sim uma fase natural da vida da mulher e muitas passam por ela sem queixas ou necessidade de medicamentos. Outras têm sintomas que variam na sua diversidade e intensidade. No entanto, em ambos os casos, é fundamental que haja, um acompanhamento sistemático visando à promoção da saúde, o diagnóstico precoce, o tratamento imediato dos agravos e a prevenção de danos (BRASIL, 2008).

O climatério é fenômeno fisiológico decorrente do esgotamento dos folículos ovarianos que ocorre em todas as mulheres de meia idade, entre os 35 e 65 anos, seguido da queda progressiva da secreção de estradiol, hipoestrogenismo progressivo e o surgimento de sintomas característicos: vasomotores, ressecamento vaginal, dispaurenia (relação sexual dolorosa) e urgência miccional, culminando com a interrupção definitiva dos ciclos menstruais (LORENZI et al., 2006).

De acordo com Lorenzi et al. (2005) as mulheres atingem valores do índice de massa corporal (IMC) mais elevados entre os 50 e 59 anos, período este concedente com a menopausa. 
O aumento do IMC pode vir acompanhado pela obesidade que é definida como aumento de depósito de triglicérides nas células adiposas. Durante o processo de envelhecimento, as mulheres sofrem alterações no perfil metabólico que modificam as composições e distribuições do tecido adiposo, o que favorece não somente o aumento ponderal, com também a progressão de eventuais processos ateroscleróticos, reconhecidamente implicados na gênese e progressão da doença cardiovascular, que, por sua vez, constitui a principal causa de mortalidade (LORENZI et al., 2005).

Indivíduos com gordura principalmente na região abdominal, ao redor da cintura, são mais propensos a desenvolverem problemas de saúde do que se a maior parte da gordura estivesse nas coxas e quadris. Isso vale mesmo se o IMC estiver na faixa considerada normal. Mulheres com uma medida da cintura acima de 88 centímetros podem ter maior risco de sofrer enfermidades do que aqueles com menor medida de cintura devido a localização da gordura (HELIO, 2011).

O hipoestrogenismo na mulher no climatério é o grande responsável pela alteração do perfil lipídico, do aumento de peso e da gordura abdominal, estando já bem estabelecida a associação positiva entre estes fatores e a doença cardiovascular (ALDRIGHI; ASAKURA, 2001).

Este estudo teve como objetivo identificar a prevalência de sobrepeso e obesidade nas mulheres que se encontram no climatério, em uma clínica especializada em saúde da mulher no município de Maringá no estado do Paraná.

\section{METODOLOGIA}

Trata-se de um estudo transversal com enfoque quantitativo, analítico. A amostra foi realizada por conveniência, composta por 100 mulheres que foram entrevistadas em uma clínica especializada em saúde da mulher no município de Maringá. O serviço realiza atendimentos ginecológicos às mulheres dos municípios pertencentes à 15ạ Regional de Saúde do estado do Paraná totalizando 30 municípios.

Foram entrevistadas mulheres na faixa etária de 45 a 69 anos, que já estavam a mais de um ano sem menstruar e que concordaram em participar da pesquisa. Excluíram-se mulheres com histerectomia parcial ou total; enfermidades psiquiátricas; e fora da faixa etária estabelecida.

Para coleta de dados foi utilizado um instrumento estruturado elaborado pelos autores, previamente testado, composto por 18 variáveis, sendo os dados referentes, ao nome, estado civil, escolaridade, raça, ocupação, idade que ocorreu a menopausa, reposição hormonal, 
paridade, se realiza atividade física, hábitos de fumar, hábitos de bebidas alcoólicas, como considera a saúde, possui alguma comorbidade, peso, estatura, circunferência da cintura.

Para complementar o diagnóstico nutricional da mulher foi utilizado a medida da circunferência da cintura. Este indicador correlaciona-se com o IMC e avalia o tecido adiposo visceral (BRASIL, 2008).

Com relação aos dados antropométricos o peso foi aferido em balança antoprométrica com capacidade de pesar até $150 \mathrm{~kg}$ e com régua métrica de 1,00 a 1,90 metros. As mulheres foram orientadas a retirar os calçados, utilizando roupas leves. Quanto a circunferência da cintura foi aferido tendo como princípio o parâmetro de dois dedos acima do umbigo, utilizado fita métrica com escala de 1 a $150 \mathrm{~cm}$.

O diagnóstico nutricional em mulheres adulta é realizado pelo profissional de saúde que deve calcular o IMC que é o peso $(\mathrm{Kg})$ dividido pela altura em $\left(\mathrm{m}^{2}\right)$. O IMC é reconhecido pela Organização Mundial de Saúde (OMS) como a principal referência para classificação das diferentes faixas de peso. Para o cálculo do IMC utilizou-se como ponto de corte o índice < 18,5 classificado como de baixo peso; IMC de 18,6 a 24,9 classificado como de peso adequado; 25,0 a 29,9 de IMC classificado como sobrepeso e $>30,0$ a classificado como obesidade (WHO, 2011).

O projeto obteve a autorização do Centro de Capacitação e Permanente em Saúde (CECAPS) ofício nำ729/2011 da Secretaria Municipal de Saúde do município de Maringá. As mulheres foram convidadas a participar do estudo e todas as informações de esclarecimento foram oferecidas para posterior obtenção do Termo de Consentimento Livre e Esclarecidas (TCLE) resolução no 466/2012 devidamente autorizada e assinada, só assim os dados foram coletados. Foi submetido e autorizado pelo Comitê Permanente de Ética em Pesquisa do Cesumar (COPEC) sobre o no 013/2011.

\section{RESULTADOS}

Participaram dessa pesquisa 100 mulheres, com idade de 45 a 69 anos. De acordo com os dados obtidos, a maior prevalência de idade de mulheres que foram entrevistadas foi de 56 a 60 anos sendo 30\% da população estudada. Quanto a variável estado civil, 74\% das mulheres eram casadas, $15 \%$ viúvas e $11 \%$ estavam distribuídas entre solteiras, com união consensual e divorciadas.

Em relação à escolaridade, 30\% eram analfabetas, 38\% das mulheres possuíam o ensino fundamental, $24 \%$ com ensino médio. Oito mulheres não responderam a esta variável. Com 
relação a quantidade de filhos, $75 \%$ das mulheres entrevistadas tiveram de um a quatro filhos, $25 \%$ das mulheres tiveram de cinco a quinze filhos.

O resultado encontrado com a prática de exercícios físicos foi que apenas $38 \%$ das mulheres entrevistadas referiram praticarem exercícios físicos. Das 38 mulheres que praticam exercícios físicos, em relação a quantidade de exercícios físicos praticados por semana, 47\% (18) das mulheres realizavam exercícios físicos de quatro a sete vezes na semana, 53\% (20) das mulheres praticam de duas vezes a três vezes na semana.

Continuando com os dados das mulheres que praticam exercícios físicos, encontrou-se que $52 \%$ das mulheres praticam por um tempo de 60 minutos ao dia, $18 \%$ praticam por 30 minutos ao dia.

Em relação ao uso de bebidas alcoólicas 5\% relataram fazer uso de bebidas alcoólicas e $9 \%$ das mulheres eram fumantes. No estudo a variável não estabelecia a frequência e quantidade da ingesta alcoólica.

Levando em consideração os tipos de comorbidades referidas pelo profissional da saúde, $63 \%$ relatarem apresentarem hipertensão arterial, 17\% diabetes, $10 \%$ doença osteoarticulares e $8 \%$ relataram apresentarem doenças cardiovasculares.

No total da amostra estudada, com relação ao IMC, $42 \%$ das mulheres estavam obesas, $40 \%$ consideradas sobrepeso, $18 \%$ foram consideradas na faixa de peso adequado e nenhuma apresentou índice com baixo peso.

Considerando que a circunferência abdominal indica fatores de quantidade de gordura abdominal e relacionada com distúrbios metabólicos, mais da metade das mulheres medidas possuíam um valor (muito elevado) $\geq 88$ considerando $69 \%$ das mulheres, $18 \%$ tem medidas $\geq 80$ (elevado) e apenas $13 \%$ são $<79 \mathrm{~cm}$.

\section{DISCUSSÃO}

Os dados encontrados de analfabetismo na população estudada corroboram com estudos populacionais sobre o tema. Em 1980 eram 553 milhões, em 1990 aumentou para 560 milhões e em 2000 já somam 563 milhões. Em termos percentuais o analfabetismo feminino mundial está em queda, com valores de 38,2\% em 1980, chegando a 2000 com 26,4\%. Embora seja observado o declínio percentual do analfabetismo, esses valores ainda são altos, com uma população analfabeta mundial de 250 milhões de mulheres. O número absoluto de mulheres analfabetas, no Brasil, mantém-se estável entre 1980 e 1991, declinando em 2000. Eram, aproximadamente, 10 
milhões em 1980 e 1991, caindo para 8 milhões em 2000. Os índices, porém, são continuamente decrescente, 27,1\%, 20,2\% e 13,4\% respectivamente (AZEVEDO et al., 2002).

Encontrou-se apenas $25 \%$ das mulheres climatéricas com mais de cinco filhos isso devido a redução do tamanho da família que pode ser explicada, sobretudo, pela acentuada queda na taxa de fecundidade nas últimas três décadas, de 5,8 filhos, em 1970, chega a 1999 com 2,3 filhos. Fatores como a mudança de valores culturais do brasileiro e o ingresso maciço de mulheres no mercado de trabalho também influenciaram a redução da família ao núcleo conjugal com filhos (IBGE, 2010).

Mais da metade das mulheres entrevistadas não praticavam o exercício físico, que por sua vez, não somente aumenta a freqüência cardíaca e a oxigenação tecidual, como favorece a redução da gordura abdominal, reduzindo assim o risco cardiovascular. De acordo com Lorenzi et al. (2005), a atividade física deve ser avaliada de acordo com os critérios estabelecidos pela Sociedade Brasileira de Cardiologia (2001). Considerou-se sedentária toda mulher sem atividade física regular, ou seja, com freqüência mínima de três vezes por semana e duração inferior a 30 minutos, independente da modalidade de exercício realizado.

Dados apontam que somente $28 \%$ das mulheres americanas se exercitam mais que 3 vezes por semana e, 1 em cada 5 mulheres nunca praticou qualquer atividade física aeróbica. $O$ risco de doença cardiovascular relacionado ao sedentarismo é de 1,5 a 2,4, risco comparado a hipertensão, dislipidemia e tabagismo. Atividade física aeróbica de moderada intensidade quando realizada de forma regular, no mínimo 30 minutos ao dia pelo menos três vezes na semana pode atingir impacto na redução do risco de evento cardiovascular na ordem de 30 a 40\% (FERNANDES; PINHO; GEBARA, 2008).

De acordo com Lorenzi et al. (2005) os achados do presente estudo reforçam a teoria de que a prevalência de sobrepeso e obesidade no climatério não seria somente influenciada por fatores biológicos relacionados ao hipoestrogenismo ou do uso de terapia hormonal, mas também por fatores psicossociais e relacionados ao estilo de vida.

Atualmente, sobrepeso e obesidade devem ser considerados epidemias que atingem o mundo todo. A prevalência de indivíduos obesos na população dos Estados Unidos é de aproximadamente $35 \%$, tendo sido observado um aumento de $35 \%$ da doença na última década. Além disso, estimativas prevêem que $87 \%$ dos homens e $83 \%$ das mulheres portarão sobrepeso ou obesidade em 2015. A epidemia alastra-se também pela Europa, onde a prevalência da obesidade 
triplicou nos últimos 20 anos, acometendo $25 \%$ da população, segundo dados da OMS (CUNHA et al., 2010).

No Brasil, segundo a pesquisa de orçamento familiar de 2002-2003, realizada pelo Instituto Brasileiro de Geografia e Estatística (IBGE), 40,0\% da população apresenta excesso de peso, sendo obesos, 13,1\% das mulheres. Essa quantidade de pessoas é alarmante, uma vez que é amplamente conhecida a associação entre a obesidade e o aumento da prevalência de doenças cardiovasculares, hipertensão arterial, hipercolesterolemia, sedentarismo e diabetes. Também já foram amplamente provadas à relação obesidade e pneumopatias, desordens ortopédicas, problemas digestivos, complicações no sono, muitos tipos de câncer e problemas sociais e psicológicos (CUNHA et al., 2010).

De acordo com Fernandes et al. (2008) aproximadamente $32 \%$ da população brasileira apresenta sobrepeso (IMC > 25), sendo esta taxa de $38 \%$ para o sexo feminino, de acordo com os dados do Ministério da Saúde de 1993. A obesidade (IMC > 30) foi encontrada em $8 \%$ da população brasileira.

O Japão tem o menor IMC, de 24, para as mulheres entre os países ricos. As mulheres de Bangladesh registram o menor índice. Caso único na Europa ocidental e raro no cenário mundial é o da Itália, onde o IMC das mulheres caiu nos últimos 28 anos. Na Bélgica, Finlândia e França, o índice de massa corporal das mulheres registrou leve alto. As suíças são as mulheres mais magras da Europa, seguidas pelas francesas e italianas. O estudo recorda que o sobrepeso, ocorre pela má alimentação e da falta de atividade física, aumenta o risco de doenças cardiovasculares, diabetes, hipertensão arterial e algumas formas de câncer. O problema seria a origem de três milhões de mortes por ano (WHO, 2011).

De acordo com a literatura, o ganho de peso e a obesidade são ocorrências comuns em mulheres que estão passando pelo período do climatério, cujo ganho de peso usualmente varia entre 2 a 6 kg durante o primeiro ano desse período (FERNANDES et al., 2009). Vários estudos têm registrado que a circunferência da cintura serve como indicadores de distribuição da gordura corporal e estão associadas à ocorrência de doenças metabólicas e cardiovasculares, dentre estas a hipertensão arterial (HASSELMANN, 2008).

A gordura abdominal é reconhecida por $58 \%$ dos médicos como fator de risco significante para doença cardíaca e síndrome metabólica. No entanto, $45 \%$ dos médicos reportaram nunca terem verificado a circunferência abdominal de seus pacientes. Dados apontam que 59\% dos pacientes sob risco de doença cardíaca disseram que nunca foram informados por seus médicos 
sobre a relação entre gordura abdominal e aumento no risco desenvolver doenças cardíacas. Atualmente a maioria da população parece estar mais focada no peso do que no excesso de gordura abdominal. No Brasil $66 \%$ da população controlam seu peso na balança, comparados aos $6 \%$ que calculam o seu IMC e a 1\% que mede a circunferência abdominal (CAMPOS, 2007).

\section{CONSIDERAÇÕES FINAIS}

A partir da análise dos resultados do presente estudo, conclui-se que as mulheres do período do climatério, mostram uma situação grave em relação ao grau de escolaridade, com $30 \%$ da população entrevistada com analfabetismo.

Mais da metade das mulheres entrevistadas apresentaram o IMC acima de seus valores normais, podendo estar ligado à má alimentação e sedentarismo. Com os dados obtidos pelo IMC e circunferência da cintura é muito importante a orientação para a pratica de exercícios físicos diariamente, para diminuir os riscos de possíveis doenças cardiovasculares, sugere-se também uma alimentação saudável e equilibrada.

Com a implantação do Núcleo de Apoio a Saúde da Família (NASF) com sete equipes no município de Maringá, o profissional nutricionista está inserido e será responsável por atividades como a coordenação das ações de diagnóstico populacional da situação alimentar e nutricional; promoção da alimentação saudável para todas as fases da vida; estímulo à produção e ao consumo de alimentos saudáveis produzidos regionalmente; capacitação da ESF e participação de ações dos programas de controle e prevenção dos distúrbios nutricionais; elaboração das rotinas de atenção nutricional e atendimento para doenças relacionadas à alimentação e à nutrição, de acordo com protocolos de atenção básica.

No climatério, por incluir na vida das mulheres aspectos que abrangem modificações fisiológicas, culturais e sociais relações familiares e extra-familiares acredita-se que a abordagem interdisciplinar seria a alternativa mais completa para o atendimento à mulher climatérica. É importante o profissional enfermeiro envolver a comunidade na participação de ações visando melhoria da qualidade de vida da mesma; realizar ações de promoção de saúde, orientação alimentar saudável, prevenção do ganho de peso e consulta de enfermagem, monitorizando os dados antropométricos de peso e altura; solicitar exames complementares, avaliar os casos de riscos e quando necessário buscar o apoio especializado. Participar e coordenar atividades de educação permanente no âmbito da saúde e nutrição. 
No presente estudo, constatou-se que as políticas de atenção à saúde da mulher formuladas nacionalmente através de amplas e complexas discussões trouxeram contribuições imprescindíveis para o processo de transformação sobre o paradigma da saúde da mulher, em especial as que estão no período do climatério. Contudo, apesar dos avanços extraordinários, esse processo é dinâmico e acompanha a transformação da sociedade e, por isso, inexaurível. Portanto, a valorização da mulher, enquanto ser humano cidadã é algo destacável, e, por isso, os profissionais de enfermagem precisam atualizar-se e promover melhorias no cuidado de enfermagem à mulher no climatério.

Ainda que a extrapolação dos resultados encontrados neste estudo para a população feminina geral não seja possível, visto ser estudo realizado em um ambulatório especializado municipal, espera-se que os dados analisados e apresentados possam contribuir ou mesmo servir de referência para outras pesquisas relacionadas ao tema em questão, idealmente estudos longitudinais.

\section{REFERÊNCIAS}

Aldrighi JM, Asakura L. Gordura alimentar e climatério. Revista da Associação Médica Brasileira. 2001; 47(3):186-186. http://dx.doi.org/10.1590/\$0104-42302001000300022

Azevedo MA, Shitara A, Araujo F, Antunes MSX. Perfil do analfabetismo e do iletrismo no mundo, na América latina e caribe, no Brasil, no estado de São Paulo e no município de São Paulo. 2002.

Brasil. Ministério da Saúde, Secretaria de Atenção à Saúde, Departamento de Ações Programáticas Estratégicas Manuais de Atenção à Mulher no Climatério/Menopausa. Brasília: Editora do Ministério da Saúde, 2008. p. 192.

Campos MV. Gordura abdominal aumenta o risco de infarto. Editora de Brasília: Movimento e saúde, 2007.

Fernandes CE, Pinho JSLN, Gebara OCE. I Diretriz Brasileira sobre Prevenção de Doenças, Cardiovasculares em Mulheres Climatéricas e a Influência da Terapia de Reposição Hormonal (TRH) da Sociedade Brasileira de Cardiologia (SBC) e da Associação Brasileira do Climatério (SOBRAC). Arquivos Brasileiros de Cardiologia: São Paulo, 2008; 1:1 -23.

Fernandes RCL, Silva KS, Bonan C, Zahar SEV, Marinheiro LPF. Avaliação da cognição de mulheres no climatério com o Mini-Exame do Estado Mental e o Teste de Memória da Lista de Palavras. Cadernos de Saúde Pública. 2009; 25(9):1883-1893. http://dx.doi.org/10.1590/S0102311X2009000900003

Hasselmann MH, Faerstein, Werneck GL, Chor D, Lopes CS. Associação entre circunferência abdominal e hipertensão arterial em mulheres: Estudo Pró-Saúde. Cadernos de Saúde Pública, 2008; 24(5):1187-1191. http://dx.doi.org/10.1590/S0102-311X2008000500029 
Helio A. F. Peso e medida abdominal na cintura. 2011. [citado em 2012 ago 25]. Disponível em: http://www.copacabanarunners.net/cintura.html.

IBGE, Instituto Brasileiro de Geografia e Estatística. Mulheres escolarizadas são mães mais tarde e tem menos filhos. Sistema de Indicadores Sociais, 2010.

Lorenzi DRS, Baracat EC, Saciloto B, Padilha JR I. Fatores associados à qualidade de vida após menopausa. Revista Brasileira de Ginecologia e Obstetrícia. 2006; 312-317.

Lorenzi DRS, Danelon C, Saciloto B, Padilha Jr I. Fatores indicadores da sintomatologia climatérica. Revista Brasileira de Ginecologia e Obstetrícia. 2005; 12-19.

OMS. Organização Mundial de Saúde. Aumenta o Consumo de Bebidas Alcoólicas no Brasil, 2011. 